\title{
Interactive comment on "Assessing ecohydrological separation in a northern mixed forest biome using stable isotopes" by Jenna R. Snelgrove et al.
}

Jenna R. Snelgrove et al.

jbuttle@trentu.ca

Received and published: 11 February 2021

Response to reviewer comments

Reviewer 1 The study by Snelgrove et al. investigates if ecohydrologic separation was possible in a northern mixed forest in Ontario, Canada. Their study design is built to assess the co-evolution of mobile-, bulk soil- and xylem water isotopic compositions during the year 2016. They formulate two questions to be considered during their investigation: 1 . What are the temporal changes in the isotopic composition of soil water and xylem sap throughout the growing season, and is this behavior unique for each species? 2. Is there evidence for hydrological separation? If so, does that differ 
between species? While I think this is an important and well thought and carried out study, I have some concerns:

The discussion is very long and hard to read. While I appreciate the detail, especially by using a review- like approach to discuss the results, I feel the main message is buried under too much detail. I would suggest the authors try and cut the discussion to half the length and keep their focus on the data they worked with, or try and combine Results and Discussion for the first two points (i.e. 4.1 and 4.2) and add the review part (i.e. 4.3) as a discussion/Conclusion section. The reader would benefit a great deal and it would separate the review section clearer from the discussion. For example, the first discussion point addresses the temporal changes in isotopic composition in both soil and xylem. While the authors are doing a good job in describing data from relevant publications, they repeat some the results (e.g. L291ff, L308ff, 336ff) and fail to provide a solid interpretation, which makes this section seem unstructured and not to the point.I understand the question to be answered with this section was a "what"-question, thus indicating a descriptive answer, but the whole manuscript would benefit in my opinion, from a "why"-question, which the authors then later try to provide with the third part of the discussion (i.e. 4.3) in a review like form. I encourage the authors to try and restructure the discussion to one (or another) of the above mentioned forms.

Also, I would encourage the authors to move away from trying to prove the ecohydrological separation idea wrong and move towards a solid interpretation of their data (i.e. what causes the offsets between xylem and soil water, and therewith also include a plant focused perspective (i.e. fractionation during water uptake? Fractionation during water transport? Interaction with stored water domains?) much like they tried in the last point of the discussion. That would enable them to formulate clear and concise questions and recommendations for future investigations.

Printer-friendly version

Response: We appreciate the Reviewer's suggestions. We propose to modify the Discussion to reduce its length and to focus on the key findings of our study. As the Reviewer suggests, this can be addressed in part by removing the repetition of results 
that they noted. We will also remove the material related to the use of best-fit regression lines for the xylem water samples in dual isotope space to estimate the isotopic composition of the source precipitation from the Methods, Results and Discussion. As the reviewer notes (and as is demonstrated in the Benettin et al. HESS 2018 paper that they drew our attention to), there are serious concerns regarding this approach to estimating the source precipitation isotopic composition. We propose to include a short section in the Discussion (based on the Benettin et al. evaporation lines shown in their calculations) indicating that evaporation of xylem water through the tree bark cannot be excluded as a potential cause of our xylem water isotopic composition results.

Specific comments: ABSTRACT: Include one or two sentences about the most likely explanation at the end of the abstract (if the word count does not allow this, maybe cut one of the introductory sentences).

Response: This change will be made.

INTRODUCTION: L37 include Brooks et al. 2010 with the mentioning of the "two water worlds" hypothesis. Their publication introduces the idea before McDonells et al. 2014 publication.

Response: This change will be made.

STUDY AREA AND METHODS 2.3 L104 were the same trees cored five times? How did you manage to extract five cores from the same height? Please elaborate.

Response: Yes, the same trees were cored five times within a small vertical range (a few $\mathrm{cm}$ ) at breast height diameter. We will provide information on how this was done in the revised version of the paper.

2.4 L115 How often were these samples taken? Please add. Also, how long were the Printer-friendly version samples stored in Ziploc bags before measurements? Please discuss the concerns raised by Herbstritt et al. (2014) and Hendry et al. (2015) regarding potential water losses using ziplog bags in this context.

Discussion paper

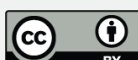


Response: This information was provided in the original submission (soil samples were taken concurrent with the xylem water samples - line 113). Samples were stored for no more than 2 weeks prior to analysis. The Hendry et al. (2015) paper suggests that water losses should therefore not be an issue. We will note this in the revised version of the paper.

L118 how far away were the lysimeters to the trees cored for xylem sap? Why did you not use trees close to the lysimeters? Also, make sure you use the word tensiometer or lysimeter or suction cup consistently when talking about the mobile water fraction throughout the manuscript.

Response: This was an error in the original submission - in fact, the lysimeters were placed adjacent to trees sampled from xylem water. This will be corrected in the revised version of the paper. We meant to use "tension lysimeter" consistently throughout the manuscript, and will check to ensure that this is the case.

L126 the url does not work. Also, this section reads incomprehensible, please try to clarify.

Response: The url will be corrected (a space had been inadvertently inserted). The sentence will be reworded in the revised version of the paper.

2.5 For all three water pools (bulk soil, mobile, xylem) different methods for water extractions and measurements were used. Why is that? The data seem complicated enough and a common method would at least provide the same methodological artifact for all three pools. Please elaborate.

Response: We will include references to our previous work that shows that the direct equilibrium method for extracting bulk soil water gives similar results to cryogenic extraction for the type of soils that we examined (e.g. low clay content, low organic matter content). Further, the same instrument was used to measure the isotopic composition of the mobile and bulk soil water samples. We will also note in the revised version of 
the paper that the ICOS instrument was cross correlated with the IRMS.

L159 Please read and discuss the Benettin et al. (2018) publication in this context.

They provide solid concerns about best fit regression analysis of samples with regard to the LMWL. The implications could change the interpretations of your results, please also check in folloing sections of the manuscript.

Response: We appreciate that the Reviewer drew our attention to this paper. As noted above, we will remove the material related to the use of best-fit regression lines for the xylem water samples in dual isotope space to estimate the isotopic composition of the source precipitation from the Methods, Results and Discussion. Instead, we will include a short section in the Discussion (based on the Benettin et al. evaporation lines shown in their calculations) indicating that evaporation of xylem water through the tree bark cannot be excluded as a potential cause of our xylem water isotopic composition results.

RESULTS L204 $\mathrm{ff}$ and Fig.3b) please indicate if the samples plotting to the right of the LMWL are bulk soil water samples from the summer (expected high evaporation fractionation) or not.

Response: We propose to include a new panel in Figure 3 to indicate that most bulk soil water samples plotting to the right of the LMWL were taken in mid-summer.

L210 please discuss this in relation to the different extraction/measuremtn techniques

Response: The same instrument was used to measure the isotopic composition of the mobile and bulk soil water samples. As note earlier, the ICOS instrument was cross correlated with the IRMS.

L213 if the bulk soil samples was collected in $5 \mathrm{~cm}$ increments as indicated in MM 2.4 Printer-friendly version why not compare the soil data from $5-15 \mathrm{~cm}$ instead of $0-15$ ? Isooptic enrichment is expected to be highest in the upmost soil layers, creating a negative lc-excess.

Discussion paper

Response: We thank the Reviewer to pointing this out. We will revise Figure $3 c$ to 
compare the mobile soil water samples taken at 10 and $30 \mathrm{~cm}$ depths with bulk soil water samples from $5-10 \mathrm{~cm}$ and $10-15 \mathrm{~cm}$ and $25-30 \mathrm{~cm}$ and $30-35 \mathrm{~cm}$ depths (we have done this, and the revision does not alter our conclusions).

Fig. 4 I find the combination of different colours and symbols is confusing. If I understand the figure right, neither would be necessary since facets were used to indicate different sampling timepoints. I suggest using one colour and symbol and then differentiating with solid and unfilled symbols. Also, please make sure that the axes have the same range and tickmarks. And I think one could benefit from a vertical line indicating a 0 lc-excess.

Response: We will revise Figure 4 along the lines suggested by the Reviewer.

Generally, when printing the figures, the y axis title is not printed. I don't know if that's due to the figure resolution or format, but it might be worth checking.

Response: We are not sure why this might have occurred. This may have been an issue arising from how the pdf of our manuscript was generated and exactly what software is used for viewing. We note that Reviewer 2 did not comment on this issue.

DISCUSSION: I don't have specific comments for the discussion at this point. Please consider my suggestions above or if you can find a better solution, that's also great. I would be happy to read the manuscript again.

Response: As we noted earlier, we will revise the Discussion along the lines suggested by the Reviewer in order to reduce its length and improve its focus.

References used in this review: Benettin P, Volkmann THM, Freyberg $\mathrm{J}$ von, Frentress J, Penna D, Dawson TE, and Kirchner JW 2018. Effects of climatic seasonality on the isotopic composition of evaporating soil waters. Hydrol. Earth Syst. Sci. 22: 2881-2890. Hendry MJ, Schmeling E, Wassenaar LI, Barbour SL, and Pratt D 2015. Determining the stable isotope composition of pore water from saturated and unsaturated zone core: Improvements to the direct vapour equilibration laser spectrometry

Printer-friendly version

Discussion paper 
method. Hydrol. Earth Syst. Sci. 19: 4427-4440.

Herbstritt B, Limprecht M, Gralher B, and Weiler M 2014. Effects of soil properties on the apparent water-vapor isotope equilibrium fractionation: Implications for the headspace equilibrium method., p. Albert-Ludwigs-Univ. Freiburg i. Breisgau.

Interactive comment on Hydrol. Earth Syst. Sci. Discuss., https://doi.org/10.5194/hess-2020-

Interactive

comment 592, 2020. 